Spatial Vision, Vol. 13, No. 1, pp. 67-86 (2000).

(C) VSP 2000.

This formatted manuscript was created by the second author and does not correspond exactly to the manuscript published in the journal. References to page numbers should refer to the published article.

\title{
Perceived motion in complementary afterimages: Verification of a neural network theory
}

\author{
HYUNGJUN KIM and GREGORY FRANCIS* \\ Purdue University, Department of Psychological Sciences \\ 1364 Psychological Sciences Building \\ West Lafayette, IN 47907-1364, USA
}

Received 26 March 1999; revised 29 July 1999; accepted 29 July 1999

\begin{abstract}
Steady fixation of a regular pattern like a bar grating or concentric circles leads to a complementary afterimage at pattern offset. The afterimage has the appearance of shimmering lines that are locally orthogonal to the orientations of the inducing image. Additionally, the afterimage includes motion running parallel to the orientation of the afterimage lines. We argue that this afterimage motion supports the existence of a cue to motion that is based on the spatial organization of oriented responses. This cue was previously proposed after analysis of a neural network model of visual perception. We test predictions of the model on various types of complementary afterimage inducing stimuli. When a contrast or size gradient is included in the inducing image, the afterimage motion moves toward the higher part of the gradient, in agreement with the model. Implications of this cue for computational and neurophysiological theories of motion perception are discussed.
\end{abstract}

Key words: Aftereffect, area MT, motion direction, neural networks

\section{Introduction}

Fixating on the vertical bar grating in Figure 1 for approximately thirty seconds and then looking at a blank sheet of paper reveals an afterimage of shimmering wavy horizontal lines. The afterimage shape consists of lines that are locally orthogonal to the inducing stimulus contours. Thus, fixation of vertical lines leads to an afterimage of horizontal lines and viceversa; and fixation of concentric circles produces an afterimage of radial lines, and vice-versa. Wade (1996) credits Purkinje (1823) as the first to note this type of afterimage, and it was

*To whom correspondence should be addressed. E-mail: gfrancis@psych.purdue.edu 


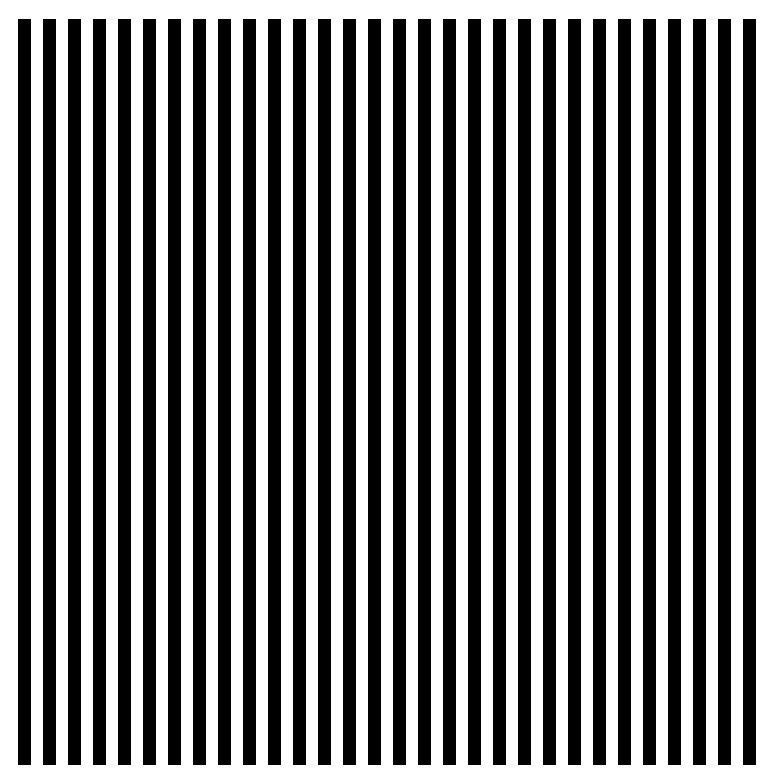

Figure 1: Fixating on this bar grating for 20-30 s and then saccading to a blank surface, reveals an orientational afterimage of shimmering horizontal lines. The lines seem to move horizontally.

rediscovered several times later (Pierce, 1900; Hunter, 1915; MacKay, 1957). MacKay suggested the afterimage be called a complementary afterimage (CAI), as the afterimage shape is an orthogonal complement of the inducing image contours.

In addition to its shape, the CAI also includes a streaming motion percept, which can sometimes also be seen during image fixation. The motion direction is orthogonal to the orientation of the inducing contours, thus, the motion direction perceived after fixating Figure 1 is horizontal (left or right). Fixation of horizontal bars produces vertical CAI motion. Fixation of concentric circles produces CAI motion that seems to expand from, or contract to, the center of the circles. Fixation of radial lines (or arcs) produces CAI motion that seems to rotate in either the clockwise or counterclockwise direction.

Studies of CAIs predated the seminal discoveries by Hubel and Wiesel $(1962 ; 1968)$ that cortical cells were strongly tuned to oriented edges, and it is now generally believed that the physiology underlying the shape of the CAI is related to competition and disinhibition between cells of orthogonal orientational sensitivities (Grossberg, 1980; Schwartz, 1980; Francis \& Grossberg, 1996; Vidyasagar, Buzás, Kisvárday \& Eysel, 1999). To make the explanation more concrete, Figure 2 schematizes one type of neural circuit that has been proposed to account for the shape of CAIs (Francis \& Grossberg, 1996). Separate pathways sensitive to the same position in visual space but perpendicular orientations compete via reciprocal inhibition from lower levels to higher levels. Feeding this competition are inputs gated by habituative transmitters. 


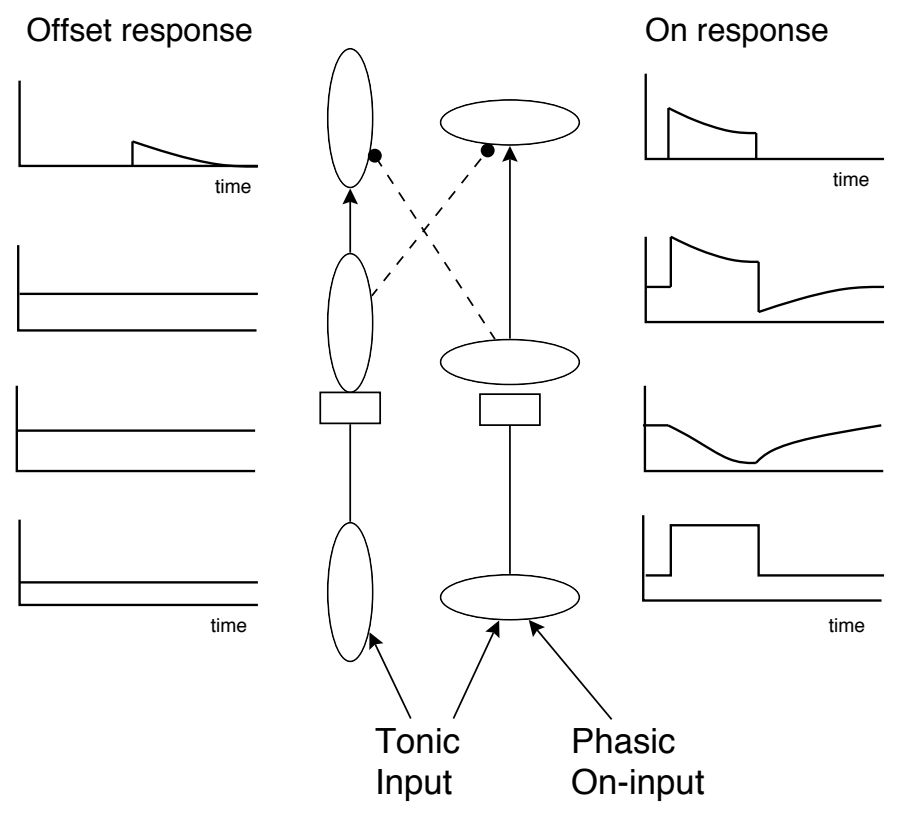

Figure 2: At stimulus offset, a gated dipole circuit produces a transient rebound of activity in the non-stimulated opponent pathway. When the pathways code opposite orientations, offset of a horizontal input leads to a rebound of vertical activity. Dashed lines with circle terminators indicate inhibition, solid arrows indicate excitation, boxes indicate transmitter gates. The plot next to each cell or gate schematizes the signal strength over time as a horizontal input is applied and removed. Offset of the horizontal input leads to a rebound of activity in the vertical pathway.

Along with signals from external stimuli, each input pathway receives a tonic source of activity, and all output signals are rectified. This combination of rectification, opponent competition, habituative transmitter gates, and tonic input creates a gated dipole circuit (Grossberg, 1972). At the offset of stimulation, a gated dipole circuit generates a transient rebound of activity in the previously non-stimulated pathway.

The time plot next to each cell or gate describes the dynamics of this circuit. In the case shown, the sharp increase and then decrease of the time plot at the lower right of Figure 2 indicates that an external input stimulates the horizontal pathway. In response to the stronger signal being sent to the next level, the amount of transmitter in the gate inactivates, or habituates, during stimulation and then rises back toward the baseline level upon stimulus offset. Notice that the inactivation and reactivation of transmitter occurs more slowly than changes in the activities of the neural cells. Each slowly habituating transmitter multiplies, or gates, the more rapidly varying signal in its pathway, thereby yielding net overshoots and undershoots at input onset and offset, respectively. During stimulation, the horizontal channel wins the 
rectified opponent competition against the vertical channel as indicated in the top right time plot. However, upon offset of the stimulation to the horizontal channel, the input signal returns to the baseline level but the horizontal transmitter gate remains habituated below its baseline value. As a result, shortly after stimulus offset, the gated tonic input in the horizontal channel has a net signal below the baseline level. Meanwhile, the vertical pathway maintains the baseline response at all cells and gates before the opponent competition. Thus, when the horizontal channel is below the baseline activity, after stimulus offset, the vertical channel wins the rectified opponent competition and produces a rebound of activity as shown in the top left time plot. As the horizontal transmitter gate recovers from its habituated state, the rebound signal in the vertical channel weakens and finally disappears. Francis and Grossberg (1996) showed how these orientational rebounds generated at offset of CAI inducing images could group together to form CAIs.

Francis and Grossberg (1996) also offered a computational purpose for these types of afterimages. In their model of visual processing, a system called the Boundary Contour System (BCS) uses a large set of individual neurons that are linked by excitatory and inhibitory connections to promote computations necessary for visual perception. Grossberg and Mingolla (1985) showed that the excitatory feedback improves the network's ability to process spatial information. However, the benefits of excitatory feedback for spatial processing come at a cost for temporal processing. The activity generated by excitatory feedback loops dominates the temporal properties of the BCS and simulations in Francis, Grossberg and Mingolla (1994) demonstrated that, if left unchecked, resonating signals could last for hundreds of simulated ms. To prevent resonating signals from lasting indefinitely, a gated dipole for orientations was introduced into the system, and the response of the orthogonal orientation at contour offset acted as a reset signal that inhibited the activities supported by excitatory feedback.

The properties of reset signals explain why visible persistence of static stimuli varies inversely with stimulus luminance and duration (e.g., Bowen, Pola \& Matin, 1974), why the persistence of illusory contours is greater and differently affected by stimulus duration than luminance contours (Meyer and Ming, 1988), why orientation adaptation effects persistence of oriented gratings (Meyer, Lawson \& Cohen, 1975), and why persistence is directly related to spatial frequency (Meyer \& Maguire, 1977). In each case, stimuli that produce strong responses among oriented cells feeding into gated dipoles generate a strong reset signal at stimulus offset. The strong reset signal greatly reduces persistence. Weaker stimuli (smaller luminance, shorter duration, fewer luminance contours, or higher spatial frequency) produce weaker reset signals at stimulus offset, thereby allowing persisting neural signals to last longer. Details of these model properties are in Francis et al. (1994) and Francis (1999).

A recent neurophysiological study of macaque monkeys is consistent with the behavior of gated dipole circuits tuned for orientation. Ringach, Hawken and Shapley (1997) explored dynamic changes in the orientation tuning curves of cells. They measured cell action potentials during rapid presentation of a series of sine gratings with different orientations. They then correlated action potentials with the grating orientations at successive times before each action 


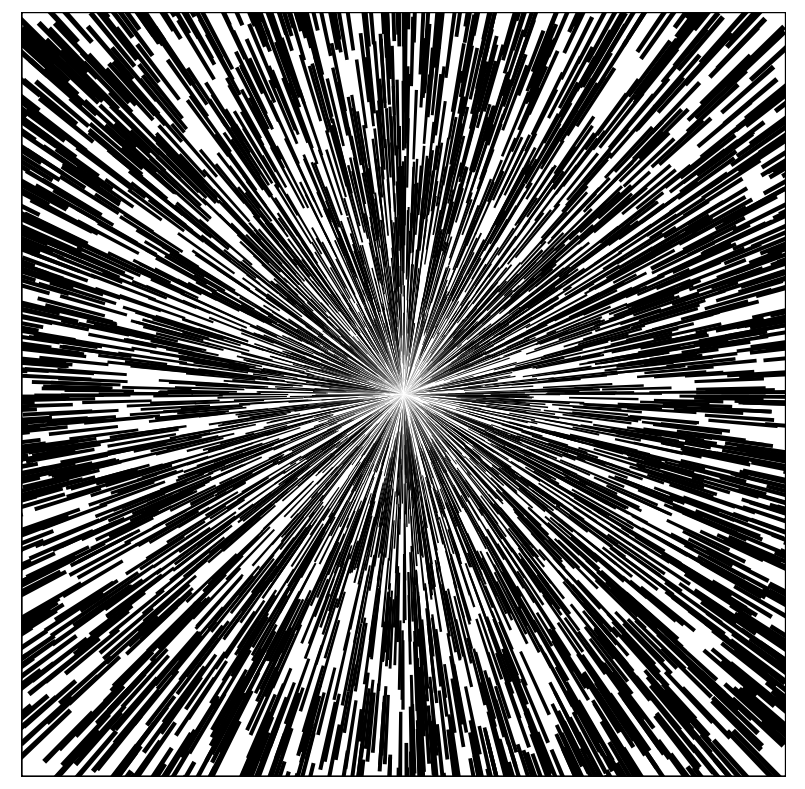

Figure 3: A CAI inducing image that does not generate noticeable shimmer during fixation, but does produce a strong CAI after a saccade to a blank surface. The CAI consists of shimmering lines organized circularly and rotational motion. Reprinted from Fermüller et al. (1997).

potential. They found that action potentials correlated with one orientation after a short delay were also correlated with the orthogonal orientation after a longer delay. This finding is consistent with the properties of gated dipoles. The top vertically oriented cell in Figure 2 is sensitive to the presentation of a vertical edge, but it also responds to the offset of a horizontal edge. The delay between the horizontal edge offset and the vertical cell response corresponds to the time needed for the rebound signal to develop.

While there is evidence from computational theories and neurophysiological studies that the shape of CAIs is related to rebounds among cells sensitive to the (local) orthogonal orientation of the inducing stimulus, attempts to explain CAI motion have not been as successful. While oriented cells in striate cortex are often tuned to motion (Hubel \& Wiesel, 1968), their motion preference is nearly always orthogonal to their orientation preference (Albright, 1984). This is in contrast to the CAI, which gives a motion percept parallel to the perceived orientation of the afterimage lines. Thus, a neurophysiological explanation must turn to other types of cells to account for CAI motion. Pierce (1900) suggested that CAI motion was a true motion aftereffect that was based on the illusory movement or shimmer often perceived while viewing these types of images (Gregory, 1993; Fermüller, Pless, \& Aloimonos, 1997). This theory cannot be the whole story, however, because numerous images do not produce the shimmering percept but do create a moving CAI. Figure 3 (reproduced from Fermüller, et al., 1997) does not produce 
(a)

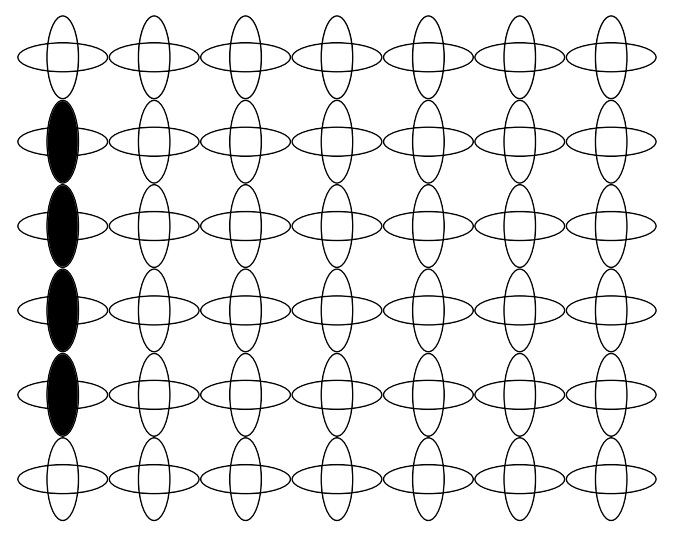

(b)

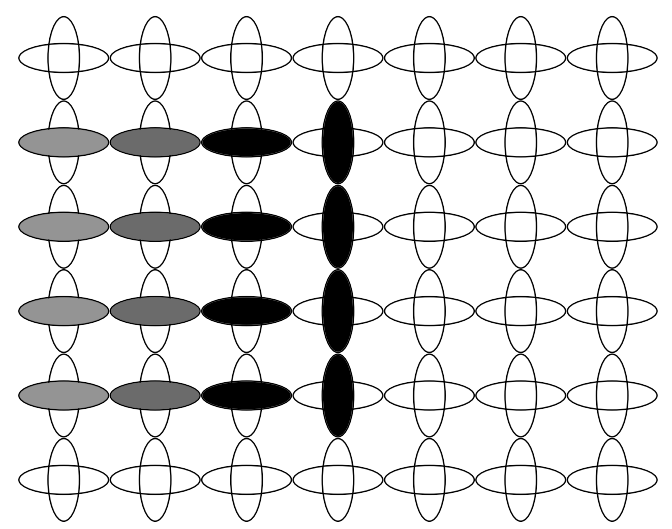

(c)

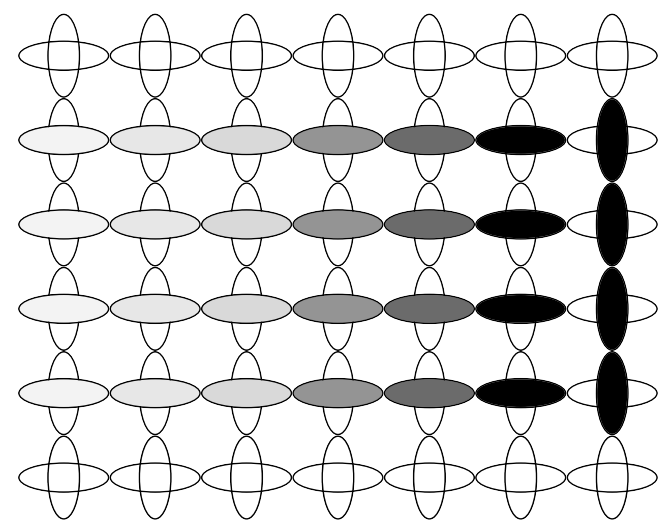

Figure 4: Hypothesized behavior of orientationally tuned cortical cells in response to a moving vertical bar. (a) At the start of the movement, the bar excites a column of vertically tuned cells. (b) As the bar moves, the gated dipole competition between orientations generates a trail of horizontal activities. (c) As the bar moves further, the beginning parts of the trail grow weaker, thus creating a gradient of horizontal activities. We hypothesize that the visual system is tuned to the properties of this trail to identify stimulus motion, direction, and speed. 
notable shimmer during fixation, yet an orientational afterimage with rotational motion can be observed after a saccade to a blank sheet of paper. Likewise, neither a retinal explanation (Taylor, 1958) nor an explanation hypothesizing disinhibition between pattern and motion channels (Georgeson, 1976) has withstood experimental scrutiny (MacKay, 1961; MacKay \& MacKay, 1976).

We suggest that motion in CAIs demonstrates a cue to motion that was first predicted by Kim and Francis (1998) after analysis of the BCS model. Consider a vertical bar moving from left to right in the visual field. In the model, such a bar should produce gated dipole rebounds among horizontal cells along its movement path, as schematized in Figure 4. Such a trail of orientational rebounds contains information about the bar's movement. For example, the relative strength of responses within a trail provides information about the direction of motion. The strongest horizontal responses will be closest to the stimulus, with weaker responses near where the bar started moving (Figure 4c). Likewise, the speed of a moving bar is partly coded by the length of its rebound trail, with faster stimuli leaving longer trails. These properties were simulated by Kim and Francis (1998) and they proposed that the visual system is sensitive to the properties of rebound trails as indicators of motion direction and speed. In particular, responses of horizontally tuned cells along a horizontal line should be a cue that something has moved horizontally. The gradient of response strength along the line will indicate the direction of the movement, and the length of the line will give a relative indication of the movement speed.

Kim and Francis (1998) noted that a technique used by artists to indicate movement in drawn images is consistent with the suggestion that rebound trails act as cues to motion. Motion lines (also called action lines or speed lines) give impressions of motion in static images (Carello, Rosenblum \& Grosofsky, 1986). We now suggest that the motion in CAIs is due to the presence of the same cue to motion. The offset of a vertical bar grating produces a CAI with horizontal responses, and those responses will be lined up horizontally, much like the responses created when a vertical edge moves across the field of view. Thus, the pattern of oriented responses in a CAI can act as a cue to horizontal motion. Likewise, a set of concentric circles gives rise to a radial CAI, which acts as a cue to either motion expansion or contraction. For every inducing image, the proposed motion cue correctly predicts the perceived axis of motion. The following experiments demonstrate that CAI motion direction can be controlled by designing CAI inducing images that produce a gradient of oriented rebound strengths. The perceived CAI motion is always in the direction of the gradient increase.

\section{Experiment 1: Contrast}

This experiment demonstrates how the perceived direction of motion in a CAI is controlled by mechanisms that modify the relative strengths of the CAI and thereby predict motion in specific directions. We generated vertical and horizontal gratings whose bars varied in contrast against 
(a)

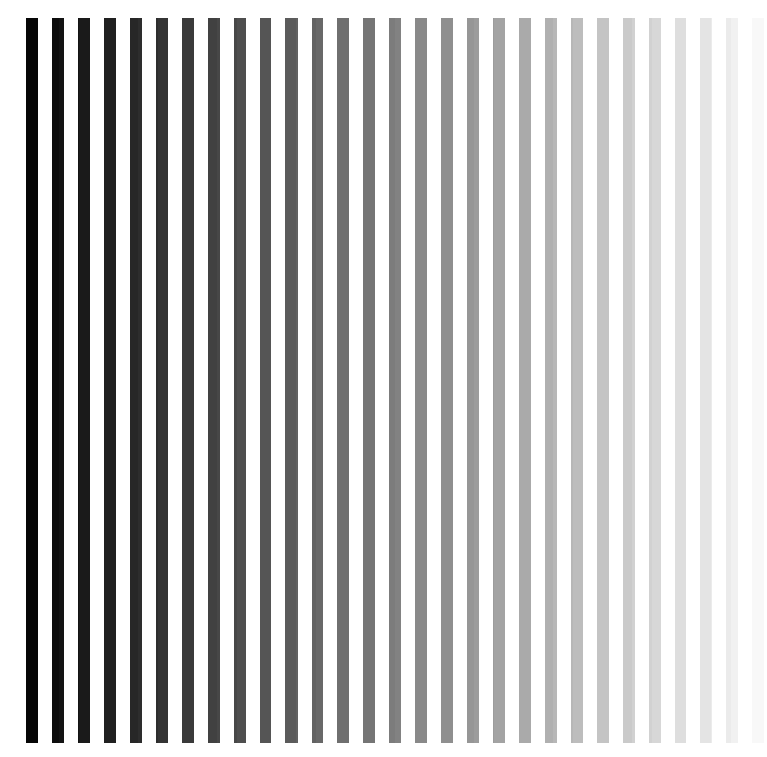

(b)

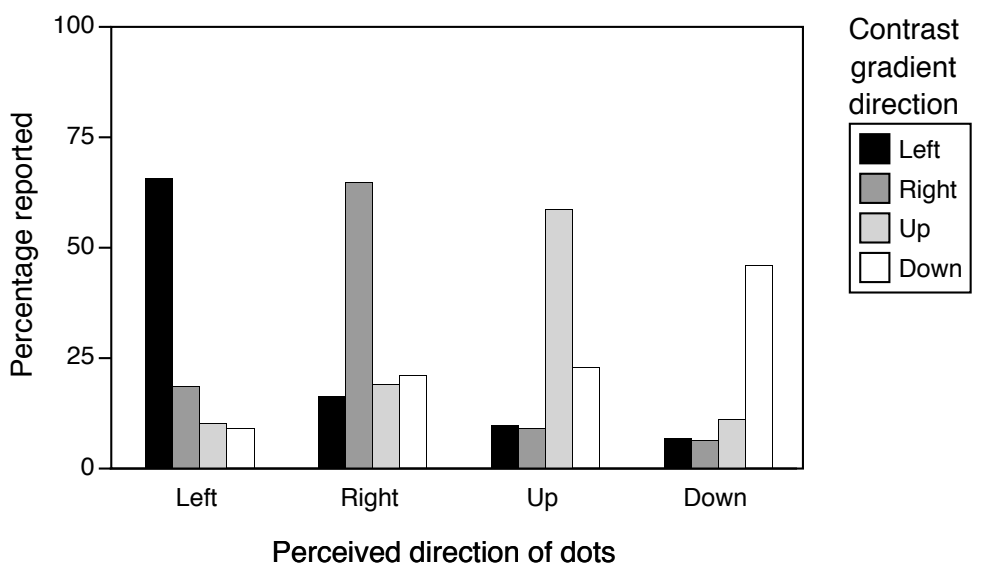

Figure 5: Experiment 1. (a) An example of the inducing stimuli in experiment 1. Contrast was varied across bars to produce a gradient of rebound strengths. (b) The percentage of time observers reported seeing the dots move in the given direction for bar gratings with between-bar contrast in the given direction. The theory predicts that observers should report seeing dot movement in the same direction as the between-bar contrast, and the data verify this prediction. 


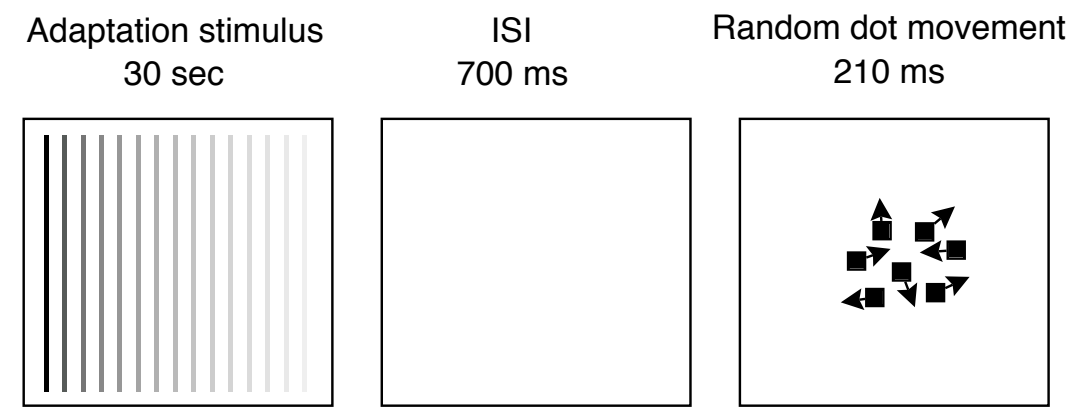

Figure 6: Schematic of the experimental procedure for all experiments. Every trial consisted of $30 \mathrm{~s}$ adaptation to the inducing image, followed by random dot movements. The observer's task was to judge the dominant direction of movement among the dots.

a white background, as schematized in Figure 5a for a vertical grating. At the offset of such a grating, the gated-dipole mechanism predicts that the bars of higher contrast should produce more habituation and stronger orthogonal rebounds. Thus, offset of the grating in Figure 5a will produce a CAI with a right-to-left increasing gradient of responses. Such a gradient of rebound signals should act as a cue to motion to the left, so the theory predicts that such a CAI will have an accompanying percept of motion to the left.

\section{$2.1 \quad$ Method}

Figure 6 schematizes the experimental method used in each trial for all experiments. The observer fixated a small gray square at the center of a CAI inducing stimulus for $30 \mathrm{~s}$. Seven hundred ms after stimulus offset, randomly moving dots were presented for $210 \mathrm{~ms}$. Pilot studies indicated that the CAI motion captured the perceived motion of the random dots. The observer's task was to indicate the perceived dominant direction of movement among the dots.

Three observers, naive to the purpose of the study and with normal or corrected-to-normal vision, participated in two testing sessions for each of four adaptation stimuli (vertical or horizontal grating and two contrast directions for each grating type).

All stimuli were presented on a Silicon Graphics Indy computer. The adaptation gratings consisted of 84 vertical or horizontal gray bars on a white $\left(52 \mathrm{~cd} / \mathrm{m}^{2}\right)$ background. The darkest bar had a luminance of $0.06 \mathrm{~cd} / \mathrm{m}^{2}$ and the brightest bar had a luminance of $47 \mathrm{~cd} / \mathrm{m}^{2}$; intermediate bars had equally spaced HSB values relative to the two extremes. The adapting grating was square shaped, with edges filling 25 degrees. Each bar was 7 arcmin thick, with a separation between bars of 15 arcmin. The subsequently presented 200 random dots were restricted to a 13 degree square filling the center of the monitor. Each dot was a small square subtending 7 arcmin on each side, and was gray $\left(41 \mathrm{~cd} / \mathrm{m}^{2}\right)$ on white. A dot changed position from one frame to the next, moving 15 arcmin in a vertical and/or horizontal direction, with 
frame duration being approximately $30 \mathrm{~ms}$. Experimental trials were blocked by adaptation stimulus, so that an observer saw only one adaptation stimulus during a testing session. During that session, the observer made 32 judgments on the perceived direction of the random dots and 8 judgments for each of the four directed movement conditions (described below). The different dot conditions were randomly mixed during a session.

\section{$2.2 \quad$ Procedure}

On half of the trials all dots moved in random directions from one frame to the next. The other trials were catch trials, where $25 \%$ of the dots (different dots for each pair of frames) moved in a fixed direction along one of the major axes, while $75 \%$ of the dots moved randomly. The observer's task was to report the perceived direction of movement among the presented dots. The choices were restricted to up, down, left, or right, and observers were asked to make their best judgment if none of these choices were clearly applicable. After making their choice with a keypress, the observer started the next trial with another keypress.

\subsection{Results and discussion}

Figure 5b plots the percentages of reported directions, on the random dot direction trials, for adaptation gratings with different orientations and directions of low to high contrast. The theory predicts that perceived direction should match grating adaptation contrast direction, and the data support this prediction. All observers showed the same pattern of results, so the data were averaged across observers in Figure 5b and all subsequent figures. There were similar but weaker effects for the catch trials, where observers tended to report motion directions consistent with the dots that moved nonrandomly. The results are consistent with the proposed claim that a gradient of oriented responses is a cue to motion in the direction of the gradient increase.

\section{Experiment 2: Control}

Experiment 2 was designed to exclude an alternative hypothesis for the results of experiment 1. It is conceivable that the results were caused by contrast only, rather than the strength of rebounds engendered by the contrast gradient. Although we do not know of any motion theory that would predict afterimage motion based solely on the direction of a contrast gradient, it seemed prudent to explore the possibility that afterimage motion was controlled by a contrast gradient alone. Thus, we generated a vertical adaptation grating whose contrast varied within each bar, as schematized in Figure 7a. If CAI motion percepts are controlled by the direction of contrast, the stimulus will produce a CAI with upward movement. If CAI motion is caused by a response gradient in the CAI, as proposed, then the CAI should include horizontal motion, 
(a)

(b)
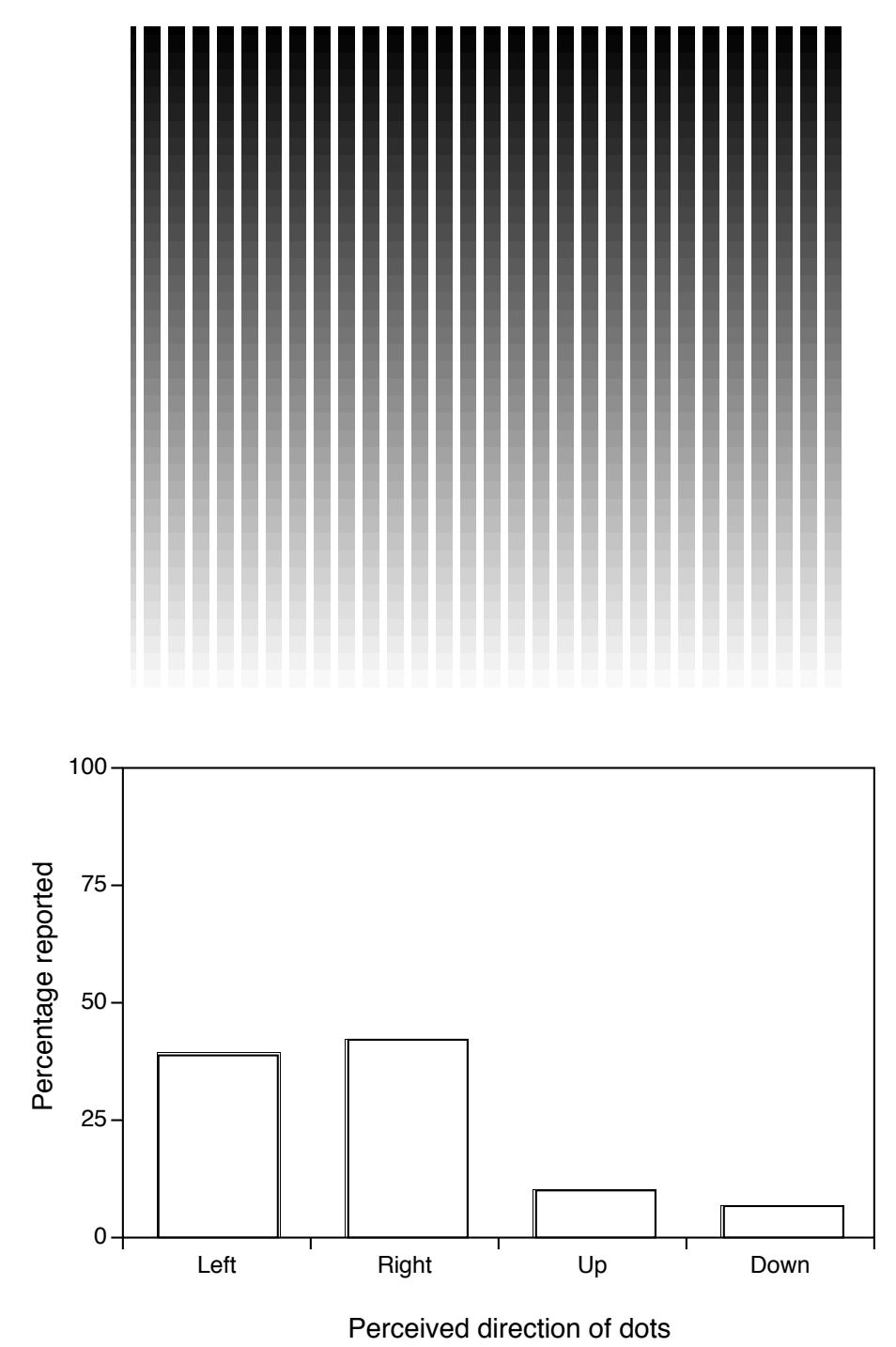

Figure 7: Experiment 2. (a) The inducing image varied contrast within each bar. (b) The percentage of time observers reported seeing the dots move in the given direction. The theory predicts that CAI motion (and thus the perceived motion of the dots) should be horizontal, and the data verify this prediction. 
with observers reporting left or right motion due to random variations in habituation or dot movements.

Three observers participated in this experiment, one of the authors (HK) and two others who were naive to the purpose of the study. All had normal or corrected-to-normal vision. The same observers participated in all subsequent experiments. The stimuli and procedures in the second experiment were the same as in the first, except that the contrast of the grating was varied within a bar, as schematized in Figure 7a. Since every grating type showed the predicted effect in experiment 1, only a vertical bar grating, with highest contrast from top to bottom, was used in experiment 2. Each observer participated in one experimental session.

Figure $7 \mathrm{~b}$ plots the percentages of reported directions, on the random dot direction trials. Observers rarely reported vertical motion, and instead reported motion to the left or right. The contrast gradient does not determine motion direction in the CAI, except when it produces a gradient in the CAI strength as in experiment 1 . The results are consistent with the theory.

\section{Experiment 3: Bar thickness}

Experiment 1 demonstrated that a gradient of CAI responses provides a convincing cue to motion direction. This finding is consistent with the proposed gated dipole mechanism that underlies the CAI. The gated dipole mechanism has also been used (Francis, et al., 1994; Francis, 1999) to explain properties of visual persistence. In this theory, persistence is the results of neural excitatory feedback loops that could keep neural responses active long after stimulus offset. Gated dipole rebounds at stimulus offset provide a source of inhibition that curtails such persisting signals. Thus, stimulus properties that lead to stronger rebounds in the gated dipole should both decrease visual persistence and be capable of generating a gradient of CAI responses. This relationship is already demonstrated by the effect of contrast in experiment 1 and the known inverse-intensity properties of visual persistence (Bowen et al., 1973; Francis et al., 1994). Visual persistence duration is also effected by stimulus size, or bar grating spatial frequency, with gratings of high frequency having longer persistence than gratings of low frequency (Meyer \& Maguire, 1977). Francis (1999) analyzed gated dipole rebounds in the BCS and noted that oriented responses were stronger for gratings of lower frequency and thus the gated dipole rebounds were stronger for low frequency stimuli. Applying this observation to CAIs predicts that a bar grating with a gradient of bar thicknesses should produce a corresponding gradient of rebound responses. The CAI should then include a percept of motion direction toward the location of the largest bar. Experiment 3 tests this prediction.

The methods were similar to experiment 1, except an adaptation stimulus similar to the one in Figure $8 \mathrm{a}$ was used. The 28 bars were all black $\left(0.06 \mathrm{~cd} / \mathrm{m}^{2}\right)$ on a white background (52 $\left.\mathrm{cd} / \mathrm{m}^{2}\right)$. The largest bar had a thickness of 56 arcmin; and the thinnest bar had a thickness of 3 arcmin. Intermediate bars had thicknesses linearly related to the extremes. The white space between bars was held constant at 28 arcmin. According to the theory, such a stimulus should 
(a)

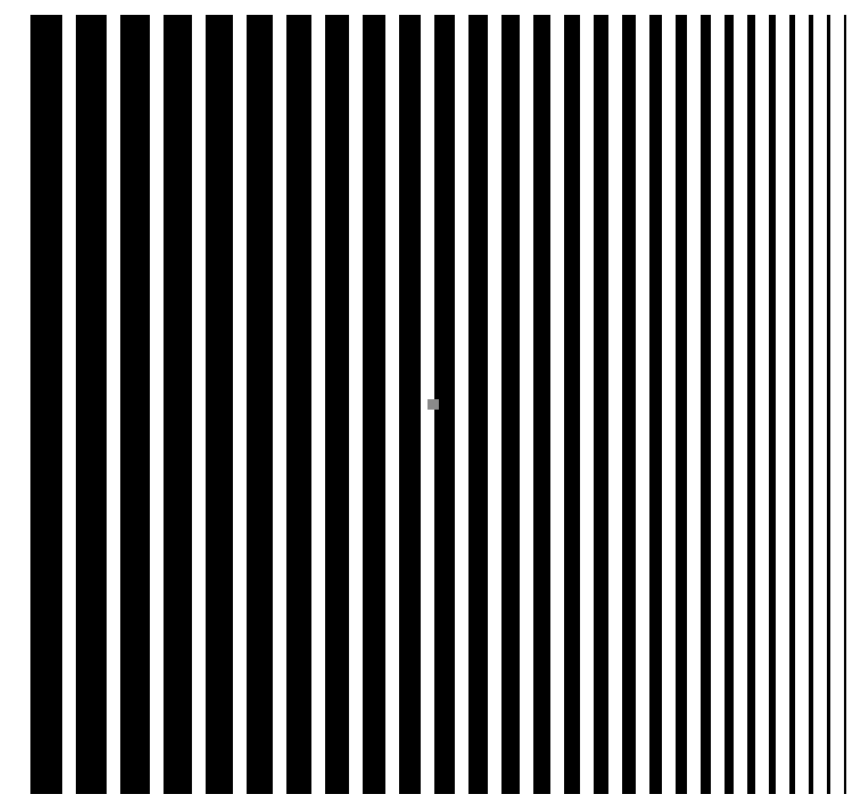

(b)

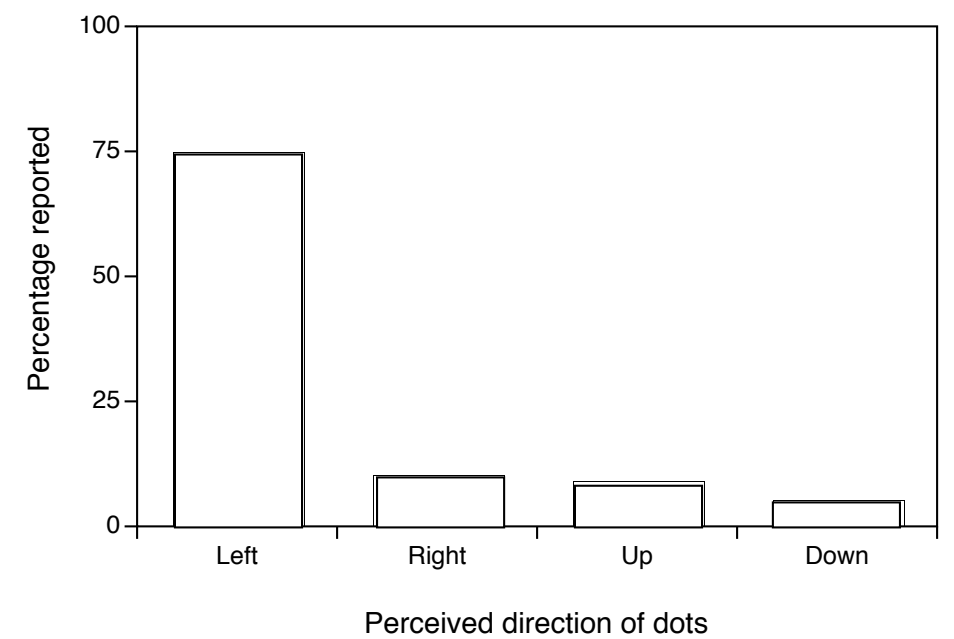

Figure 8: Experiment 3. (a) The inducing image decreased bar thickness from left to right. (b) The percentage of time observers reported seeing the dots move in the given direction. The theory predicts that observers should report seeing dot movement in the same direction as the thickest bar (left), and the data verify this prediction. 
produce a CAI with leftward motion. Each observer participated in one experimental session.

Figure $8 \mathrm{~b}$ plots the percentages of reported directions, on the random dot direction trials. Observers reported leftward motion on $72 \%$ of the trials, which supports the prediction that CAI motion direction is influenced by a gradient of bar thicknesses. The data support the dual role of gated dipoles in CAIs and control of visual persistence. The effects of bar thickness on CAI motion direction provides independent support for the analysis in Francis (1999) that gated dipoles, in the BCS theory, produce stronger rebounds for larger bars.

The CAI produced by the changing thickness bar grating was quite strong, and all observers further reported that CAI motion to the left was visible even without the random dots.

\section{Experiment 4: Circular inducers, contrast}

Experiments 4 and 5 were variations of experiments 1 and 3, but used concentric circles as the CAI inducing stimuli instead of bar gratings. Circle inducers produce a strong radially organized CAI with either inward or outward motion. We wanted to be certain that the effects of luminance and bar thickness found earlier generalized to another type of CAI inducing stimulus. Experiment 4 varied the contrast to bias motion direction and experiment 5 varied circle thickness.

The methods were nearly identical to those in Experiment 1, except only two types of inducers were used and the choices of perceived motion were modified. The inducers consisted of 32 concentric circles (same thickness and extreme luminances as the bars in experiment 1). Circle luminances were set to either predict inward motion (highest contrast at the innermost circle) or outward motion (highest contrast at the outermost circle). Figure 9a shows the inducing stimulus for an outward contrast gradient. A fixation point was not used, instead observers fixated on the innermost circle.

The catch trials in the random dot displays were limited to leftward or rightward movements only, and the observer was asked to choose between motion percepts of left, right, in, or out. Observers participated in two experimental sessions (one for each inducing stimulus).

Figure $9 \mathrm{~b}$ plots the percentages of reported directions on the random dot direction trials. The inducer with the highest contrast on the innermost circle predominately produced CAI percepts with inward motion, while the inducer with the highest contrast on the outermost circle predominately produced CAI percepts with outward motion. These results agree with the data in experiment 1 and with the proposed theory.

\section{Experiment 5: Circle inducers, thickness}

Experiment 5 was like experiment 3, except the inducing stimulus consisted of 17 concentric circles instead of a grating of bars. The thickest circle was 37 arcmin, and the thinnest circle was 3 arcmin. The white space between circles was 28 arcmin. Figure 10a shows the inducing 
(a)

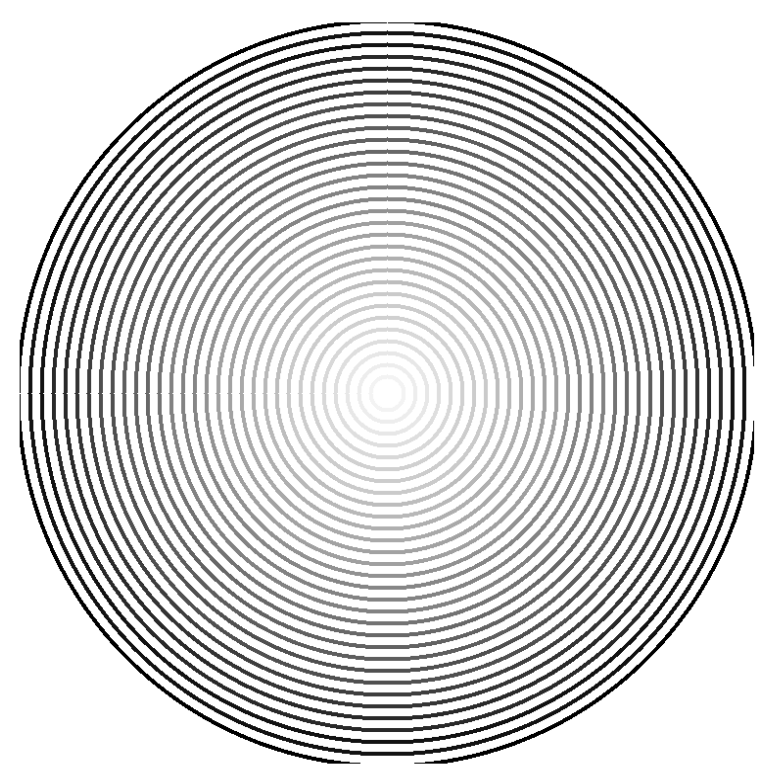

(b)

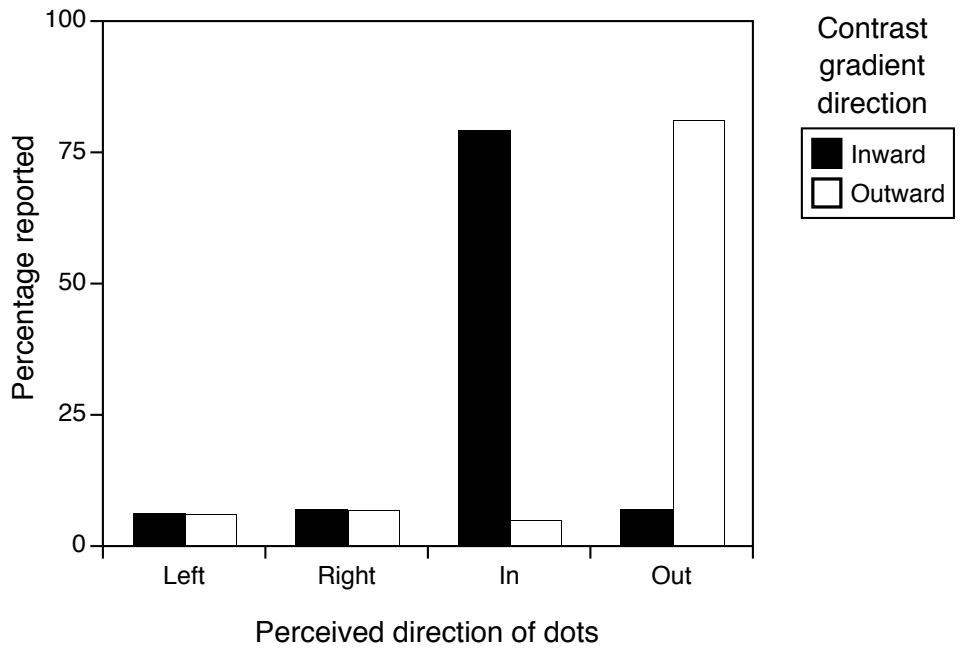

Figure 9: Experiment 4. (a) The inducing images varied contrast across circles. For this image the highest contrast is on the outermost circle. Another image placed the highest contrast on the innermost circle. (b) The percentage of time observers reported seeing the dots move in the given direction. The theory predicts that observers should report seeing dot movement in the same direction as the contrast gradient, and the data verify this prediction. 
stimulus for an inward gradient of circle thickness. The theory predicts that inducers with the largest thickness on the innermost circle (Figure 10a) will produce inward motion in the CAI, and inducers with the largest thickness on the outermost circle will produce outward motion in the CAI.

Figure 10b plots the percentages of reported directions, on the random dot direction trials. Observers predominately reported inward and outward motion as predicted. Thus, experiments 4 and 5 demonstrate that the effects of contrast and bar thickness found in experiments 1 and 3 generalize to another type of inducing stimulus.

\section{Conclusions}

A conclusion from these experiments is that some part of the visual system is tuned to recognize an oriented pattern of oriented cell responses as a cue to motion. That contrast and bar thickness both control perceived direction of motion in the CAI provides strong evidence that a gated dipole mechanism is related to both CAIs and properties of visual persistence (Francis \& Grossberg, 1996).

Neural mechanisms sensitive to these orientational cues as indicators of motion may fit into theories of motion processing in area MT (Albright, 1984; Movshon, Adelson, Gizzi \& Newsome, 1986; Rodman \& Albright, 1989; Simoncelli \& Heeger, 1998). Albright (1984) classified motion sensitive cells in MT as type I or II. Both types of cells showed strong orientation selectivity. Type I cells also showed motion selectivity orthogonal to the preferred orientation. Type II cells showed motion selectivity parallel to the preferred orientation. The properties of type II cells are consistent with the notion that CAI trailing afterimages act as a cue to motion. A type II cell sensitive to this cue will be driven by either a stationary bar parallel to the cell's preferred motion direction, or by movement of an orthogonal bar or spot moving in the preferred direction and thereby leaving a trail of oriented rebounds that are of the cell's preferred orientation.

Additional research (Rodman \& Albright, 1989) found that type II cells were essentially the same as the pattern-motion cells noted by Movshon et al. (1986). These pattern-motion cells seemed to respond to the overall pattern of a plaid grating, rather than to its individual components. Computational models of pattern-motion cells (Albright, 1984; Simoncelli \& Heeger, 1998), based largely upon intersection of constraint (IOC) calculations, have offered an explanation of the parallel relationship between motion and orientation sensitivity in type II (or pattern-motion) cells. These theories appropriately combine motion signals from local motion based on oriented filters in such a way as to calculate the global direction and speed that is consistent with all the local motions. In these types of theories, any motion occurring in a static bar must be parallel to the bar's orientation, and hence undetected by local motion signals. Thus, if a pattern-motion MT cell gives any response to an oriented static bar, it will be for a bar parallel to the cell's preferred direction of motion.

There may be difficulties applying these theories to our data. Simoncelli and Heeger (1998) 
(a)

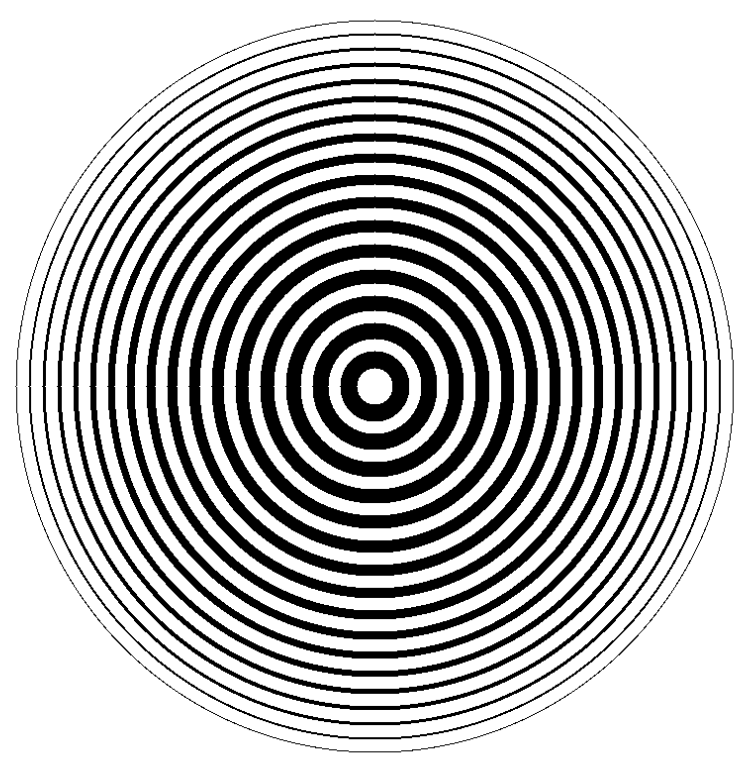

(b)

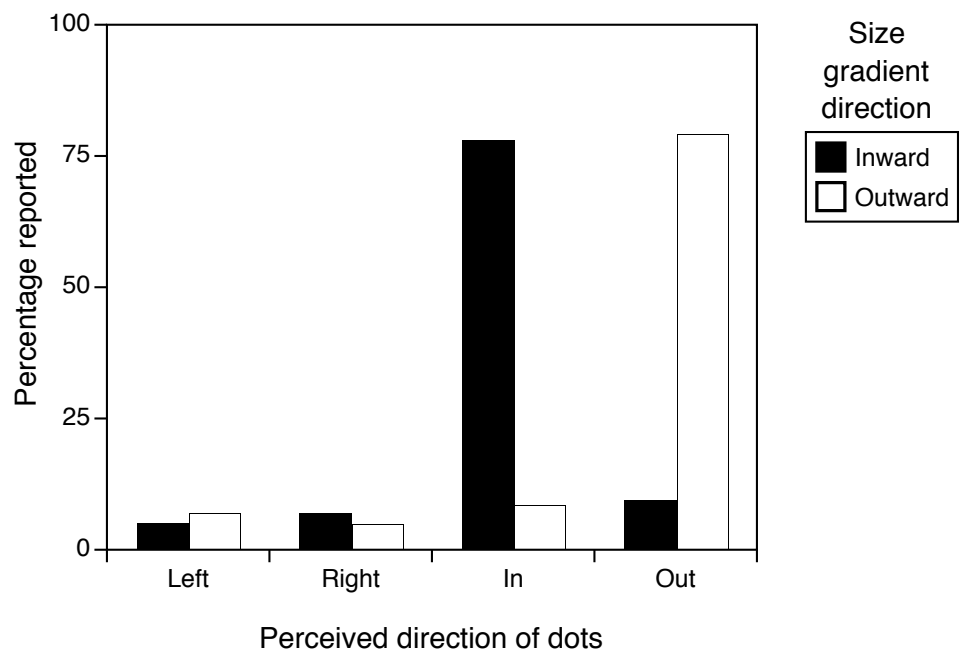

Figure 10: Experiment 5. (a) The inducing images varied circle thickness. For this image the innermost circle is thickest. For another image the outermost circle was thickest. (b) The percentage of time observers reported seeing the dots move in the given direction. The theory predicts that observers should report seeing dot movement in the same direction as the size gradient, and the data verify this prediction. 
modelled neurophysiological properties rather than visual percepts, and as they note, the representation of stimulus velocity must include a distribution of model MT responses. It is not clear that the responses of these model MT cells are easily related to the motion percept. In particular, motion is not generally perceived when a bar moves parallel to its orientation. If model cells respond to the bar as if it is moving, some additional mechanisms must frequently prevent those responses from contributing to the overall percept. In addition, motion parallel to an oriented bar does not provide any constraints for IOC types of algorithms. Thus, from a computational perspective the MT cells could interpret a static bar as moving parallel to its orientation, or not make such an interpretation, with no loss to the computation. There is little justification for assuming MT cells interpret a static bar as moving, except that it accounts for the neurophysiological properties. These models accurately account for the what, but not the why, of this specific neural behavior. Our analysis offers an explanation of why MT cells might be sensitive to static oriented bars. Namely, such bars provide the same type of information, albeit misleading information, as is generated by the rebound trail of a truly moving orthogonal bar.

The neural responses generated by a static bar are correlated with movement. If the IOC types of models are extended to account for CAI movement, then they must make some additional assumptions about how a gradient pattern of oriented responses influences MT cells. A MT pattern-motion cell sensitive to, say, upward motion should be sensitive to a static vertical bar with a contrast gradient strongest on the top (like one of the bars in Figure 7a). It should be much less responsive to a vertical bar with a contrast gradient strongest on the bottom. More generally, such a cell should respond very strongly to the CAIs in experiments $1,3,4$ and 5 .

An additional neurophysiological prediction is that the rebounding cells in V1 noted by Ringach et al. (1996) should project to the pattern-motion cells in MT. Ringach et al. (1996) found rebounding cells in layers $2,3,4 \mathrm{~B}, 5$, and 6 . Layer $4 \mathrm{~B}$ and layer 6 of $\mathrm{V} 1$ are known to project to MT (see review in Maunsell \& van Essen, 1983) so that the connection of rebounding cells to type II cells in MT is plausible. Rebounding signals could also take a different route to type II cells in MT by first projecting to area V2 and then to MT.

Our analysis is not the first to hypothesize that a moving stimulus may leave a path that indicates properties of its movement. Shepard and Zare (1983) demonstrated that an apparent motion stimulus seemed to follow a path indicated by a brief faint arc flashed during the interstimulus interval. They hypothesized that the arc was interpreted by the visual system as motion blur. More recently, Geisler (1999) hypothesized that oriented cells in visual cortex may respond to motion streaks and that these responses may code motion parallel to a cell's orientation (see also Harrington, Harrington, Wilkins and Koh; 1980). However, these approaches will require modification if they are to account for our results. The CAI afterimage is quite different from motion blur or motion streaks, as it seems to contain orientation but no noticeable brightness. Without this brightness it is difficult to see how Shepard and Zare's (1983) hypothesis could account for our findings. Geisler's quantitative model interprets the re- 
sponse of a single oriented cell to a motion streak. Such a model correctly predicts that oriented responses indicate motion along an axis parallel to their orientation, but it cannot explain why, in our experiments, motion is seen primarily in one direction along the axis. Geisler's model fails here because a single cell cannot represent a gradient of intensity across the motion streak. Our results suggest that producing a gradient of responses across a set of orientationally tuned cells is critical for controlling the direction of CAI motion. Geisler's model could be modified so that the interpretation of motion streaks occurs at a higher level of the visual system, as we have proposed.

Our analysis of CAI trails further suggests a radical rethinking of tasks dependent on motion information. For example, McBeath, Shaffer and Kaiser (1995) considered how people can position themselves to catch a fly ball in baseball. They noted that moving objects do not leave a path by which people can keep track of and predict the position of the baseball. Our analysis of CAI motion and its relationship to moving objects suggests that moving objects do leave a contour trail that indicates the trajectory of the object. Such a trail could be analyzed, in part, by mechanisms tuned solely to spatial aspects of visual perception. Investigations of optic flow may benefit by including the new cue and its influences on perception. Likewise, bar gratings are a common stimulus in a variety of studies of motion perception (e.g., Bressan, Ganis \& Vallortigara, 1993; Kim \& Wilson, 1993) and may benefit by considering how the CAI generated by such stimuli influence perceived motion directions.

We finish by addressing a potential problem with the cue. If the spatial layout of oriented cells can act as a cue to motion, then why do static contours not always seem to move in a direction parallel to the contour orientation? A possible solution reveals itself by considering a significant difference between contours generated by static images and contour trails generated by moving objects and in CAIs. The contour in a CAI does not include a percept of color or brightness, but is more akin to an awareness of contour existence. It could be argued that the CAI shape is a sort of form afterimage, without an accompanying brightness percept. This observation is consistent with the BCS model used in Kim and Francis (1998) to predict the existence of the motion cue. In this model the BCS codes contour information but does not code brightness or color information. The latter is coded in a complementary system called the Feature Contour System (FCS) (Grossberg \& Mingolla, 1985; Grossberg \& Todorović, 1988). We suggest that the extra feature signals generated by a static contour prevent the motion signals based on such contours from generating a percept of motion, even though the signals may contribute to the recognition of implied motion. The contour trail generated by a moving object should have the same properties as the contours generated by a CAI, that is, no FCS responses. Thus, details of the proposed mechanism at once support the distinctions between the BCS and FCS and suggest further interactions between the systems as they contribute to motion percepts. 


\section{References}

Albright, T. (1984). Direction and orientation selectivity of neurons in visual area MT of the Macaque. Journal of Neurophysiology, 52, 1106-1130.

Bowen, R., Pola, J., \& Matin, L. (1974). Visual persistence: Effects of flash luminance, duration and energy. Vision Research, 14, 295-303.

Bressan, P., Ganis, G., \& Vallortigara, G. (1993). The role of depth stratification in the solution of the aperture problem. Perception, 22, 215-228.

Carello, C., Rosenblum, L., \& Grosofsky, A. (1986). Static depiction of movement. Perception, 15, 41-58.

Fermüller, C., Pless, R., \& Aloimonos, Y. (1997). Families of stationary patterns producing illusory movement: insights into the visual system. Proceedings of the Royal Society of London B, 264, 795-806.

Francis, G. (1999). Spatial frequency and visual persistence: Cortical reset. Spatial Vision, 12, $31-50$.

Francis, G. \& Grossberg, S. (1996). Cortical dynamics of boundary segmentation and reset: Persistence, afterimages and residual traces. Perception, 25, 543-567.

Francis, G., Grossberg, S., \& Mingolla, E. (1994). Cortical dynamics of binding and reset: Control of visual persistence. Vision Research, 34, 1089-1104.

Geisler, W. S. (1999). Motion streaks provide a spatial code for motion direction. Nature, 400, 65-69.

Gregory, R. L. (1993). A comment: MacKay rays shimmer due to accommodation changes. Proceedings of the Royal Society of London B, 253, 123.

Georgeson, M. (1976). Antagonism between channels for pattern and movement in human vision. Nature, 259, 413-415.

Grossberg, S. (1972). A neural theory of punishment and avoidance: II. Quantitative theory. Mathematical Biosciences, 15, 253-285.

Grossberg, S. (1980). How does a brain build a cognitive code? Psychological Review, 87, 1-51.

Grossberg, S. \& Mingolla, E. (1985). Neural dynamics of perceptual grouping: Textures, boundaries, and emergent segmentations. Perception \& Psychophysics, 38, 141-171. 
Grossberg, S. \& Todorović, D. (1988). Neural dynamics of 1-D and 2-D brightness perception: A unified model of classical and recent phenomena. Perception and Psychophysics, 43, 241277.

Harrington, T. L., Harrington, M. K., Wilkins, C. A., \& Koh, Y. O. (1980). Visual orientation by motion-produced blur patterns: Detection of divergence. Perception \& Psychophysics, 28, 293-305.

Hubel, D. H. \& Wiesel, T. N. (1962). Receptive fields, binocular interaction and functional architecture in the cat's visual cortex. Journal of Physiology, 160, 106-154.

Hubel, D. H. \& Wiesel, T. N. (1968). Receptive fields and functional architecture of monkey striate cortex. Journal of Physiology, 195, 215-243.

Hunter, W. (1915). Retinal factors in visual after-movement. Psychological Review, 22, 479489.

Kim, H. \& Francis, G. (1998). A computational and perceptual account of motion lines. Perception, 27, 785-797.

Kim, J. \& Wilson, H. R. (1993). Dependence of plaid motion coherence on component grating directions. Vision Research, 33, 2479-2489.

MacKay, D. (1957). Moving visual images produced by regular stationary patterns. Nature, 180, 849-850.

MacKay, D. M. (1961). Interactive processes in visual perception. In W. A. Rosenblith (Ed.). Sensory communication (pp. 339-355). New York: MIT Press.

MacKay, D. M. \& MacKay, V. (1976). Antagonism between visual channels for pattern and movement? Nature, 263, 312-314.

Maunsell, J. H. R. \& van Essen D. C. (1983). The connections of the middle temporal visual area (MT) and their relationship to a cortical hierarchy in the macaque monkey. Journal of Neuroscience, 3, 2563-2586.

McBeath, M. K., Shaffer, D. M. \& Kaiser, M. K. (1995). How baseball outfielders determine where to run to catch fly balls. Science, 268, 569-573.

Meyer, G., Lawson, R., \& Cohen, W. (1975). The effects of orientation-specific adaptation on the duration of short-term visual storage. Vision Research, 15, 569-572.

Meyer, G. \& Maguire, W. (1977). Spatial frequency and the mediation of short-term visual storage. Science, 198, 524-525. 
Meyer, G. \& Ming, C. (1988). The visible persistence of illusory contours. Canadian Journal of Psychology, 42, 479-488.

Movshon, J. A., Adelson, E. H., Gizzi, M. S. \& Newsome, W. T. (1986). The analysis of moving visual patterns. In Chagas, C., Gattass, R. \& Gross, C. (Eds.), Pattern recognition mechanisms, (pp. 117-151). New York: Springer.

Pierce, A. H. (1900). The illusory dust drift: A curious optical phenomenon. Science, 12, 208-211.

Purkinje, J. (1823). Beobachtungen und Versuche zur Physiologie der Sinne. Beiträge zur Kenntniss des Sehens in subjectiver Hinsichts., Prague: Calve.

Ringach, D., Hawken, M. J., \& Shapley, R. (1997). Dynamics of orientation tuning in macaque primary visual cortex. Nature, 387, 281-283.

Rodman, H. R. \& Albright, T. D. (1989). Coding of visual stimulus velocity in area MT of the macaque. Vision Research, 27, 2035-2048.

Schwartz, E. (1980). Computational anatomy and functional architecture of striate cortex: A spatial mapping approach to perceptual coding. Vision Research, 20, 645-669.

Shepard, R. N. \& Zare, S. L. (1983). Path-guided apparent motion. Science, 220, 632-634.

Simoncelli, E. \& Heeger, D. (1998). A model of neuronal responses in visual area MT. Vision Research, 38, 743-761.

Taylor, W. (1958). Visual organization. Nature, 182, 29-31.

Wade, N. (1996). Descriptions of visual phenomena from Aristotle to Wheatstone. Perception, 25, 1137-1175.

Vidyasagar, T. R., Buzás, P., Kisvárday, Z. F. \& Eysel, U.T. (1999). Release from inhibition reveals the visual past. Nature, 399, 421-422. 